\title{
Vision influence on whole-body human vibration comfort levels
}

\author{
Maria Lúcia Machado Duarte* and Matheus de Brito Pereira \\ DEMEC, Mechanical Engineering Departament, UFMG, Federal University of Minas Gerais, Av. Ant ônio Carlos, \\ 6627, Pampulha, Belo Horizonte/MG, 31270-901, Brazil
}

\begin{abstract}
The well being of people needs to be a priority in the modern world. In that respect, vibration cannot be one more cause of stress. Besides that, vibration comfort is very important, since high levels may cause health or even tasks' accomplishment problems. Several parameters may influence the levels of vibration a human being supports. Among them, one can mention the influence of gender, age, corporeal mass index (CMI), temperature, humor, anxiety, hearing, posture, vision, etc. The first three parameters mentioned were already investigated in previous studies undertaken by GRAVI (Group of Acoustics and Vibration) researchers. In this paper, the influence of vision is evaluated. The main objective with this series of tests performed is to try to quantify in a future the influence of each parameter in a global vibration comfort level. Conclusions are presented for the parameter investigated.
\end{abstract}

Keywords: Human vibration comfort, vision, perception threshold, maximum acceptable vibration, and whole-body vibration

\section{Introduction}

Problems related to vibration stimulus are very frequent in people's life nowadays. Those stimuli may damage health or even disturb the activities that people perform. Several factors can be quoted that increase these problems, such as the growth of aerial traffic and the number of heavy vehicle in the cities, acting like unpleasant sources of noise and vibration. The use of metallic structures is another factor that worsen these problems since they are more susceptible to vibration problems than concrete structures due to their low damping characteristics [9].

Some people feel more the vibration stimuli than others since there are several parameters that influence the human responses to vibration. There are books [5], standards [7,8] and papers [1,4,17] related to whole-body human vibration but none of them study individually the influence of each parameter that may affect the vibration levels obtained. As mentioned in [5], most standards do not even mention which variables are known to have a large influence on response. Therefore, they do not provide the necessary information to effectively reduce the vibration effects. Moreover, the use of international standards may not be appropriate to the Brazilian reality. The familiarity to everyday vibration due to bad roads, bad transportation vehicles, the influence of climate and humor, may affect differently the results obtained for the Brazilian community, since these factors may have an influence on the levels obtained and are generally different from the communities were the standard results were obtained.

Therefore, since 2001, a series of research with the aim to study the parameters that may influence people's response in a more individual way are being carried out by GRAVI DEMEC-UFMG (Group of Acoustics and Vibration of the Federal University of Minas Gerais) researchers. The study presented here is part of this series and belongs to a wider project studying peoples' response to whole-body vertical vibration. The objective here is to verify if vision is an important parameter that affects people's responses.

Other researchers have investigated the effects of posture [11,16] and the effects of instructions [12] on the levels obtained for whole-body vibration. Although the international standard [7] does not take into consideration such

*Corresponding author. Tel.: +55 313499 5453; Fax: +55 313443 3783; E-mail: mlduarte@ dedalus.lcc.ufmg.br. 
variables, these studies concluded that they play an important whole of the results obtained and such effects should be considered.

Previous studies by the GRAVI researchers investigated the influence of gender, age, corporeal mass index (CMI) and vision in the levels obtained. In $[9,10]$, the authors thought women were, intuitively, more sensitive to vibration than men, when they looked at the curves obtained. However, when they analyzed the results using the non-parametric test of Mann-Whitney, they concluded that gender does not influence the volunteer's responses. The same conclusion was obtained statistically in $[14,16]$, although at the latter, looking at the curves for some postures, the sensitivity for men was slightly higher than for women, opposing the finds at [10]. In [15] the influence of age and corporal mass index (CMI) on volunteer's responses was investigated. The authors concluded that age has stronger weight than the corporal mass index in this case according to the non-parametric test of Mann-Whitney. Elderly people recorded higher acceleration levels than younger people for both situations analyzed. An explanation for that may be given by the fact that the reaction reduces with age making people more tolerant to the environment stimuli. Considering the corporal mass index variable, they concluded statistically that this parameter does not have an influence on the responses, since no major differences were found. This fact counteracted what was expected intuitively, since it is known that the adipose tissue has several functions within the organism like thermal isolation and protection against mechanical shocks [13]. So, in another study, the authors decided to investigate the influence of all the parameters analyzed by the time. So, they compared the influence of gender against age, CMI and vision on the comfort levels obtained [2]. They concluded that irrespective of the parameter under investigation, all curves show the same behavior, i.e., the sensitivity decrease with a frequency increase. So, independently of the parameter, the volunteers adjust small acceleration levels for lower frequencies and high acceleration levels for higher frequencies. However, the results obtained for vision were much lower than the others for the vision parameter. So, the present paper evaluates the influence of vision in a more detailed way using the same type of study applied to the previous parameters to verify its influence on the whole-body human vibration comfort levels.

This paper is organized as follows: Section 2 presents some human body resonance frequencies. Section 3 describes the methodology applied. Section 4 presents the experimental results and their discussions. A comparison with previous studies evaluating other parameters that may influence the whole-body human vibration levels is performed at this section as well. Finally, Section 5 shows the conclusions drawn in this study.

\section{Human body resonance frequency}

The human body can be considered a complex system with several degrees-of-freedom. Moreover, it does not present response linearity when exposed to vibration stimulus. Therefore, when studying the effects of vibration on people it is important to consider not only the system response but also the pathologic, physiologic and psychological effects that the vibration energy will produce in the subject [5].

There are some studies where the body is considered as a single mass and the range of resonance frequencies is found according to the position of the body and the direction of vibration $[1,20]$. When internal organs are taken into consideration, completely different results are found. The human body will have different resonance frequencies for its internal organs depending on the direction and position of the person subjected to the vibration stimulus [7]. Several studies have been undertaken with the objective to obtain the dynamic response of human organs but some difficulties were present [20]. Such difficulties can be attributed to the fact that these studies used animals, dummies or even dead bodies instead of live people. Therefore, the frequencies found through these studies differ slightly from reality but serves as an important reference source. Table 1 presents some resonance frequency values of human body organs collected from [1,20], as presented in [9].

The majority of the values presented in the above table are a range of values due to the inter-variability inherent to human beings, that is, the some organ, but in different subjects, have different resonance frequencies. Sometimes, the same organ, in the same subject, can also have different values depending on the circumstances due to the intra-variability of the results [5]. Nevertheless, the frequencies presented in Table 1 may be used to interpret results obtained during whole-body vibration studies. 
Table 1

Resonance frequencies of human body organs

\begin{tabular}{ll}
\hline Organs & Resonance Frequencies $(\mathrm{Hz})$ \\
\hline Head & 20 to 40 \\
Spinal Column & 8 \\
Chest Wall & 60 \\
Abdominal & 4 to 8 \\
Shoulders & 4 to 8 \\
Lungs & 4 to 8 \\
Hands and arms & 20 to 70 \\
Ocular globe & 60 to 90 \\
Maxilla & 100 to 200 \\
\hline
\end{tabular}

\section{Methodology}

\subsection{Experimental set-up}

The experimental set-up and methodology adopted for the present study are the same as that used in [9]. The subjects sat on a wooden chair having metallic feet. The chair had a backrest but no cushion. It was positioned over a metallic plate $(660 \times 950 \times 3 \mathrm{~mm})$, with bent edges to provide more rigidity. The plate was simply supported by four compression springs with external diameter of $15 \mathrm{~cm}$, wire diameter of $5 \mathrm{~cm}$ and height of $36 \mathrm{~cm}$. The natural frequencies of the springs/plate/chair assembly, as well as the natural frequencies of the springs/plate alone were measured in another study [3]. The first natural frequencies for both assemblies varied even though they were below the frequencies used during the present study. Only some of the upper frequencies stayed within the tested interval. The volunteer mass would certainly alter these results, although no study was undertaken in that respect. Nevertheless, the mentioned study helped understanding the amplification/attenuation of the excitation provided by the experimental setup.

The position of the chair was such that the center of gravity of the set-up (chair + subject) was, as much as possible, coincident with the geometric center of the plate. The reason for that is to have the maximum excitation in the vertical direction ( $z$-axis), besides avoiding undesirable rotational movements. However, no special apparatus was used to guarantee that. Every time the setup was assembled, there was a concern to keep it as close as possible to the previous setup, although there was no way of guarantying that. That variation however, was assumed to be smaller than the variability between the results obtained. The contact area between the plate and the chair was only at its feet. Since the chair had metallic feet, it was the way found to assure that the vibration stimulus was mostly transmitted to the body of the volunteer.

The responses were measured by a 353B34 PCB accelerometer located at the geometric center of the plate. The ISO2631/1 standard [7] recommends the measurement to be made as close as possible to the position where the vibration enters the body. However, since the tests reported here had the intention to find the levels related to dwellings and there, both sat and standing positions would be found, the point closest to the excitation was used as the reference for the measurements. The reason is that there would happen the highest levels, so providing a more conservative result. A small magnet shaker (B\&K model 4810) was used to provide the sinusoidal excitation generated by an HP 35670A analyzer. A B\&K 2706 amplifier provided the amplification of the signal sent to the shaker. Figure 1 shows one view of the experimental set-up employed.

\subsection{Sample information}

For most of the laboratory studies undertaken related to human comfort, the volunteers were gathered from an academic environment. However, care has to be taken to prevent the responses being biased. So, in order to have the sample statistically representative it has to be as random as possible, including all kind of people, irrespective of race, social class, gender, etc. One way found in [4] to achieve that was to pay people to take part in their experiments. Due to the low budget of the research presented here, that was not possible. As a result, it was not easy to find people to take part in the experiments. So, most of the volunteers for this research were selected among the individuals 


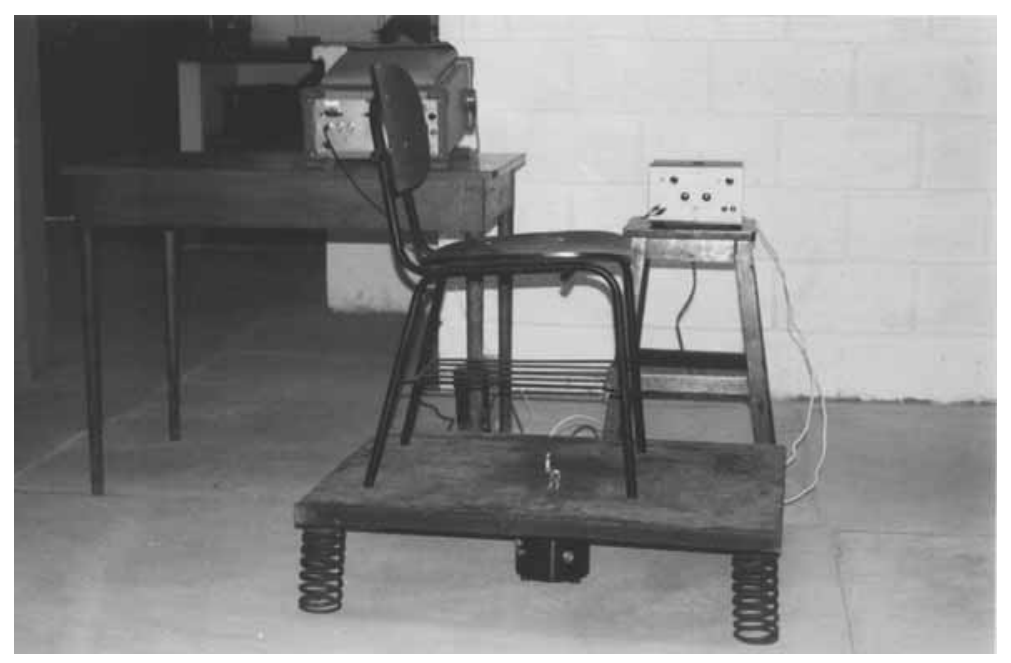

Fig. 1. Equipment set-up used.

that go to the Mechanical Engineering Department of the Federal University of Minas Gerais (where the tests were carried out) every day.

The volunteers were selected after they filled out a form about their general health condition as recommended in [5]. The researchers then gave an explanation about the experiments and another form was filled out, given their volunteers consent. In order to make the research more reliable, the Ethics Committee in Research of the University approved the project first, as that is also a requirement for researches involving human beings.

In this study, the responses from two groups of volunteers are evaluated. Each group was composed by ten subjects and represented the extremes of the parameter in study, i.e., vision. So, the subjects with their eyes covered composed one group and the subjects with uncovered eyes formed the other group. The aim is to verify if vision is an important factor that influences people responses. At this stage, only covered and uncovered eyes subjects are compared, as the use of impaired people needs a special authorization from the ethics committee of research. However, such group is being thought for the future.

The methodology adopted here, as mentioned, is the same as the one developed in [9] and so, in order to make the results comparable, the same sample size was used, i.e., 20 subjects. That sample size $(n)$ was established according to Eq. (1) [18], using a pilot sample taken as 10 subjects when investigating the parameter gender [9]. Equation (1) is based on the standard deviation $(\sigma)$ and the expected error $(E)$ obtained from the pilot sample. For a $95 \%$ confidence interval, the value of $z_{a / 2}=1.96$.

$$
n=\left[\frac{z \alpha / 2 \sigma}{E}\right]
$$

Another way of determining the sample size is to use the same size from similar experiments. In that way, most of the studies found in the literature used a sample size between 8 and 40 subjects. That validates the sample size used.

\subsection{Experiment}

The experiment described here had the objective of verifying the influence of vision on people's responses to whole-body vertical vibration considering two cases: A) the perception threshold and B) the maximum acceptable limits of vibration at residences. Despite knowing that the most common human vibration stimulus at residences is due to the fundamental resonant frequency of slab floors (that occurs normally between 4 and $10 \mathrm{~Hz}$ ), only frequencies above $16 \mathrm{~Hz}$ could be evaluated. Excitations at lower frequencies could not be applied due to the limitation of the shaker to the lowest frequency value of $16 \mathrm{~Hz}$ without causing distortion of the signal. As the standards [7,8] recommends whole-body human vibration measurements between 0 to $100 \mathrm{~Hz}$, that validates the other frequencies used. Moreover, in [5], the author mentions that excitation can be applied to floor vibration above 
$20 \mathrm{~Hz}$. The comparison among the results of each group concerning the analyzed variable was made using the Non Parametric Tests of Mann - Whitney [18].

During the test, the subjects were sat in a comfortable posture with their back upright. The adjustment method was used as explained in [5]. This method consists of asking the subjects to adjust the magnitude of the motion at each tested frequency until they reach the expected limit, according to the test case considered (that for the present study are the perception threshold and the maximum vibration limit). At the beginning of each test session, the amplifier knob was set to the initial position (i.e., position zero). The amplifier was positioned as close as possible to the subjects to prevent interfering with the experiment.

Eight frequency values were used (i.e., 16, 20, 25, 31.5, 40, 50, 63 and $80 \mathrm{~Hz}$ ) for the sinusoidal vibration at the vertical direction ( $z$-axis). These frequencies corresponded to the center frequency values of the $1 / 3$ octave band within the range from 16 to $100 \mathrm{~Hz}$ (due to the shaker limitation mentioned). Each subject responded to the vibration stimulus according to the following conditions:

- Case (A) - the lowest magnitude of vibration a subject could feel (i.e. perception threshold);

- Case (B) - the lowest magnitude of vibration that same person would consider unacceptable if it occurred in his/her residential environment (i.e. their maximum acceptable vibration limit at residences).

The subjects first made the adjustment to the perception threshold (case A) and then adjusted to the maximum acceptable vibration limit (case B). When the required level for each situation was reached, the power spectral signal was recorded on a floppy disk for future references at the same time that the RMS acceleration magnitude at the tested frequency was written onto the volunteer's test sheet. It is important to say that the frequency values were provided to the volunteers in a random way by the researcher to prevent the responses being biased.

\section{Results}

\subsection{General information}

During the experiment presented in the previous section, the temperature in the GRAVI laboratory where the tests were carried out was between $22^{\circ} \mathrm{C}$ and $30^{\circ} \mathrm{C}$. Due to the proximity of this laboratory to the workshop of the Mechanical Engineering Department, noise within the laboratory varied from around $50 \mathrm{~dB}$ (when the workshop was not functioning) to $70 \mathrm{~dB}$ (when the workshop was in operation). Although noise and temperature were observed during the tests, no consideration was made of them during the analysis of the results, since they were not measure in a consistent way.

The average time for the whole experiment (including tests and recording of the results) was around 30 minutes. The subjects could move during the recording of the results, however everybody stayed sat on the experimental set-up used, although in a more relaxed position.

\subsection{Experiment results}

Figure 2 shows the average results obtained considering covered and uncovered eyes groups together for the two studied situations investigated for the parameter vision (i.e., cases A and B). Analyzing the results, it can be noticed that for the parameter vision the participants were more sensitive in the lowest frequencies. The value of acceleration measured increased with the increase of the frequency (i.e., the sensibility decreased) until reaching a peak between $40 \mathrm{~Hz}$ and $50 \mathrm{~Hz}$ and later it decreased again. The results may have been influenced by the resonance frequencies of the head and ocular globe, as shown in Table 1. That same behavior was verified in the analysis of the parameters gender [9,10], corporal mass index (CMI) and age in previous studies [15], so indicating that, independent of the parameter under investigation, the subjects are more sensitive in lower than in upper frequencies [2], agreeing with the other researchers' results and standards $[8,14,16]$. However, these references do not show a decrease above $50 \mathrm{~Hz}$, except in [16] for the seated posture. That behavior may be attributed to the setup used in both researches $[9,16]$. It is important to stress that the experiments were carried out with the subjects seated, as the results can be influenced by the posture, orientation and position of the body when this type of study is made, as mentioned before $[11,14,16]$. 
Vision

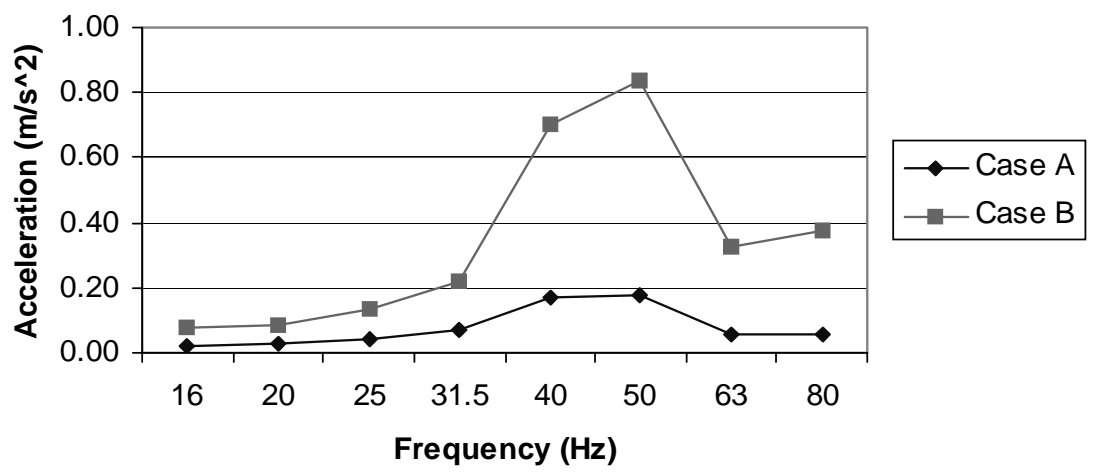

Fig. 2. Average results of the experiment considering the parameter vision (cases A and B).

Relationship B/A - Vision

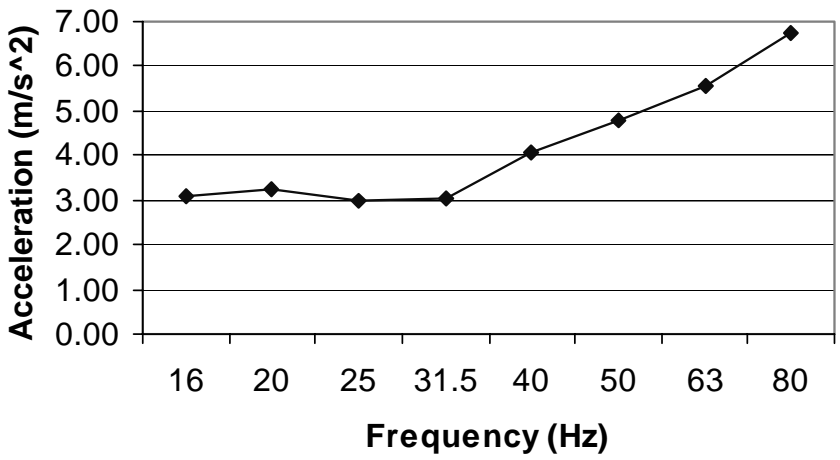

Fig. 3. Relationship among the results for cases A and B - parameter Vision.

Moreover, the setup used for the tests have influenced the results obtained, due to amplification (at low frequencies) or attenuation (at high frequencies) of the vibration stimulus [3].

Figure 3 presents the relationship B/A between the two test cases studied. The behavior of this curve is similar to that presented by the curves in the norm ISO 2631/2 [8], in other words, there is an increase in the relationship with the increase of frequency. An important observation should be made again. There is a repeatability difficulty inherent in this type of study, mainly due to the inter- and intra-subjectivity of the subjects [5].

Instead of considering all average results for the vision parameter, as presented in Figs 2, 4 and 5 shows the average results obtained for case A and case B, respectively, comparing individually each group used for the vision parameter (i.e., covered and uncovered eyes group). The scale used for the acceleration axis in each figure is intentionally different in order to stress the behavior of the curves for case A. Its levels are much lower than those obtained for case B and so, the differences between the covered and uncovered eyes groups could not be seem if the same scale for case B was used.

Visually, it can be noticed by the curves that the volunteers with covered eyes, on average, were slightly more sensitive than the volunteers with the uncovered eyes for most of the frequency values studied, registering small acceleration levels for both cases A and B. The fact of covering the eyes can leave the subjects more concentrated and aware of the vibration effects than for the uncovered eyes, so explaining their answers been smaller than for the uncovered eyes' group. It is worth to point out here that hearing could be helping them detect the vibration. However, new studies shall be made to confirm these hypotheses. 
Case A - Vision

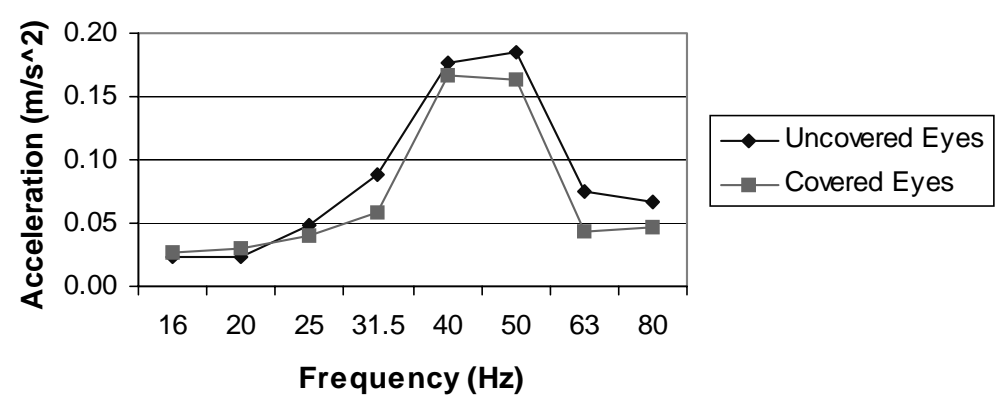

Fig. 4. Comparison among the averaged results between covered and uncovered eyes group - Case A.

Case B - Vision

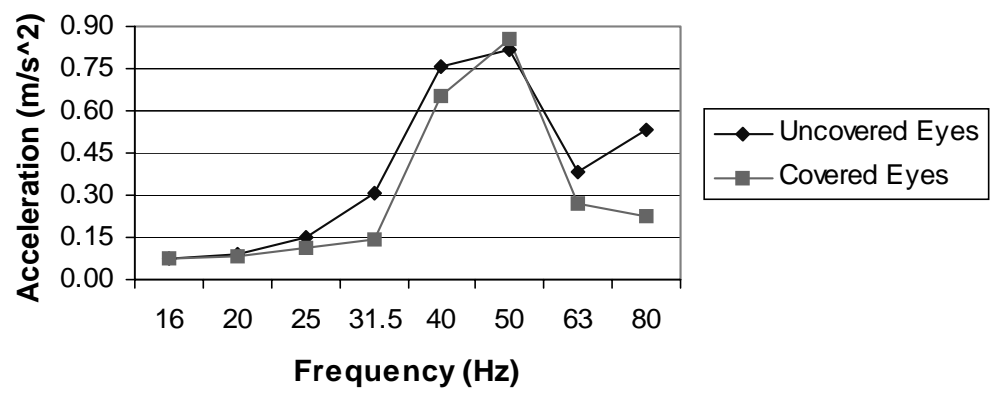

Fig. 5. Comparison among the averaged results between covered and uncovered eyes group - Case B.

Another observation has to be made with respect to the sample used for the vision study. The same volunteers were used in the two groups, however the tests were carried out at different times. This fact may have influenced the results for the second tests, once the subjects were already familiar with the experiments (i.e., they had previous knowledge of the tests). Even so, as already mentioned, there is always the subject's intra-variability, i.e., the same subject responds differently at different times [5], according to the circumstances. Therefore, in order to verify the influence of the previous knowledge influence, Figs 6 and 7 present the results' comparison at $31.5 \mathrm{~Hz}$ and $80 \mathrm{~Hz}$, respectively, for each one of the volunteers used for the two tested cases, i.e., covered and uncovered eyes. As mentioned, the results were obtained at different times. The numbering of the volunteers follows the one used since the beginning of the "Human Comfort Levels to Whole Body Vibration" study by GRAVI researchers. This explains the reason why not all volunteers' numbers are included, since for each parameter analyzed only 20 (twenty) volunteers are used, 10 (ten) for each extreme. As the volunteers for the extremes of the vision parameter were the same, only 10 volunteers are included.

Analyzing these figures, it can be noticed that for most of the volunteers there was a decrease in the answers in relation to the first test. New tests shall be accomplished to confirm if it is the vision or the previous knowledge of the test that is causing this results' decrease of most of the volunteers. It is interesting to confirm also if hearing aids the volunteers in detecting the vibration stimulus.

Table 2 presents a statistical comparison for the vision results obtained using the non-parametric tests of MannWhitney. Significant differences of $5 \%$ were not verified among the volunteers' responses at most of the frequency values considered. Only at $63 \mathrm{~Hz}$, Case A, and at $31.5 \mathrm{~Hz}$ and $63 \mathrm{~Hz}$ for case B, 5\% differences were found. This indicates that vision, statistically speaking, is not an important parameter on subject's answers.

The objective of the research where the results of this paper are part of it, is to study the influence of different parameters on the perception threshold and unacceptable levels at residences to whole-body human vibration. So, each of the graphs shown in Fig. 8 analyzed the influence of the parameter under investigation considering the 
Table 2

Statistical comparison for the variable vision

\begin{tabular}{ccccccccc}
\hline Frequency $(\mathrm{Hz})$ & 16 & 20 & 25 & 31.5 & 40 & 50 & 63 & 80 \\
\hline Case A & - & - & - & - & - & - & $*$ & - \\
Case B & - & - & - & $*$ & - & - & $*$ & - \\
\hline
\end{tabular}

- There were not significant differences between the answers of uncovered and covered eyes.

$* 5 \%$ of difference $(\mathrm{Z} \alpha / 2=1.96)$.

Vision $-31.5 \mathrm{~Hz}$

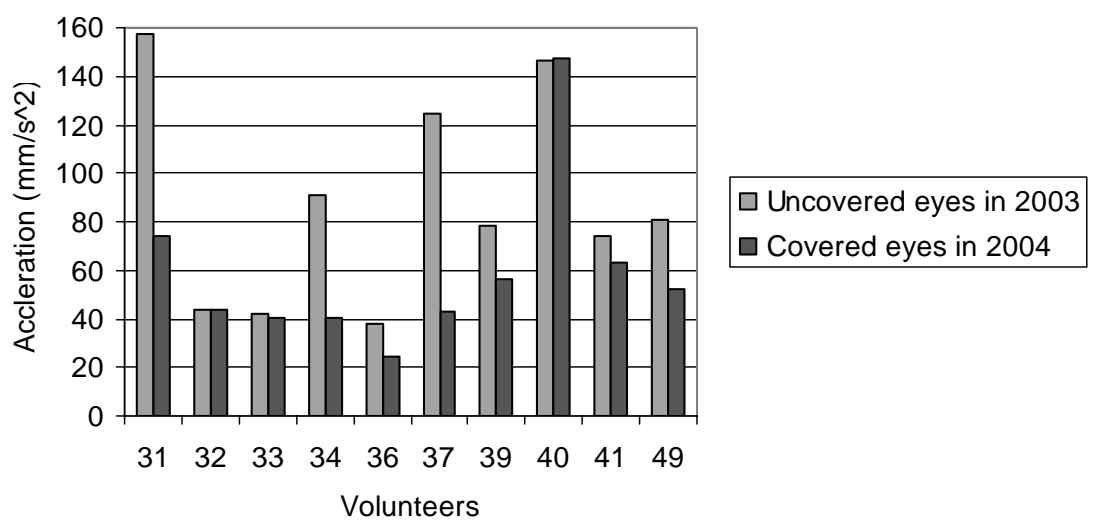

Fig. 6. Volunteers' results comparison between each vision situation studied $-31.5 \mathrm{~Hz}$.

Vision - $80 \mathrm{~Hz}$

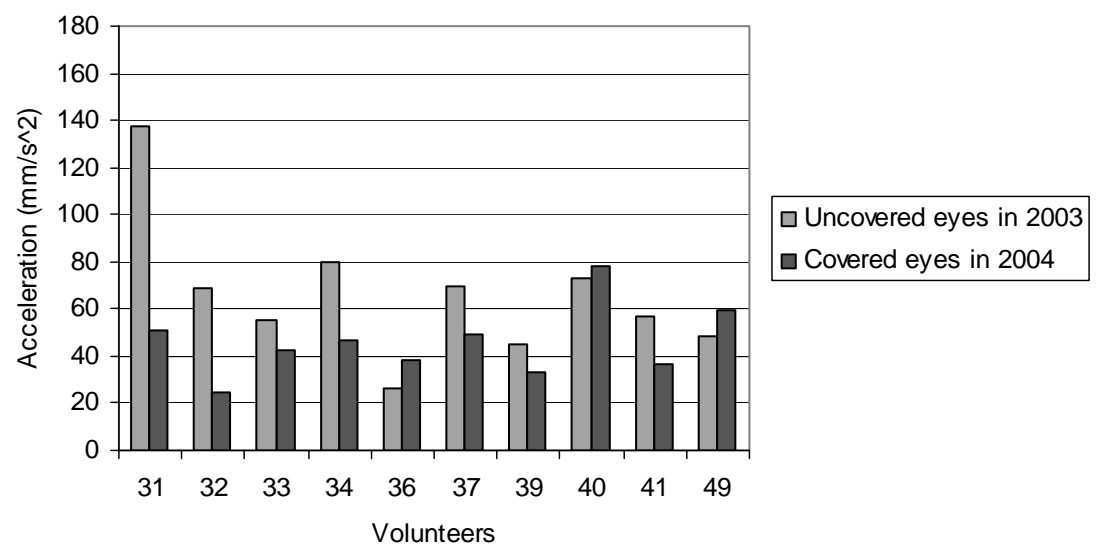

Fig. 7. Volunteers' results comparison between each vision situation studied $-80 \mathrm{~Hz}$.

average results for each of them. Basically, the difference was the volunteers used in each study. The sample for each case tried to make the weighing of each parameter different, so to verify which has the strongest influence. Therefore, this figure compares the influence of vision against the influence of age, corporeal mass index (CMI) and gender. The average results for the parameter under investigation are considered and not the average extremes. All graphs are presented using the same scale to easy the comparison.

It can be seem when analyzing this figure that vision results are much lower than the other averaged results, mainly at the least sensitive frequencies (i.e., above $40 \mathrm{~Hz}$ ). Nevertheless, as mentioned previously, all parameter 
Gender

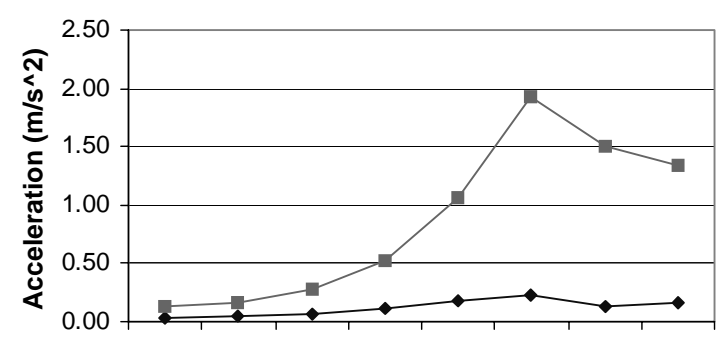

Frequency $(\mathrm{Hz})$
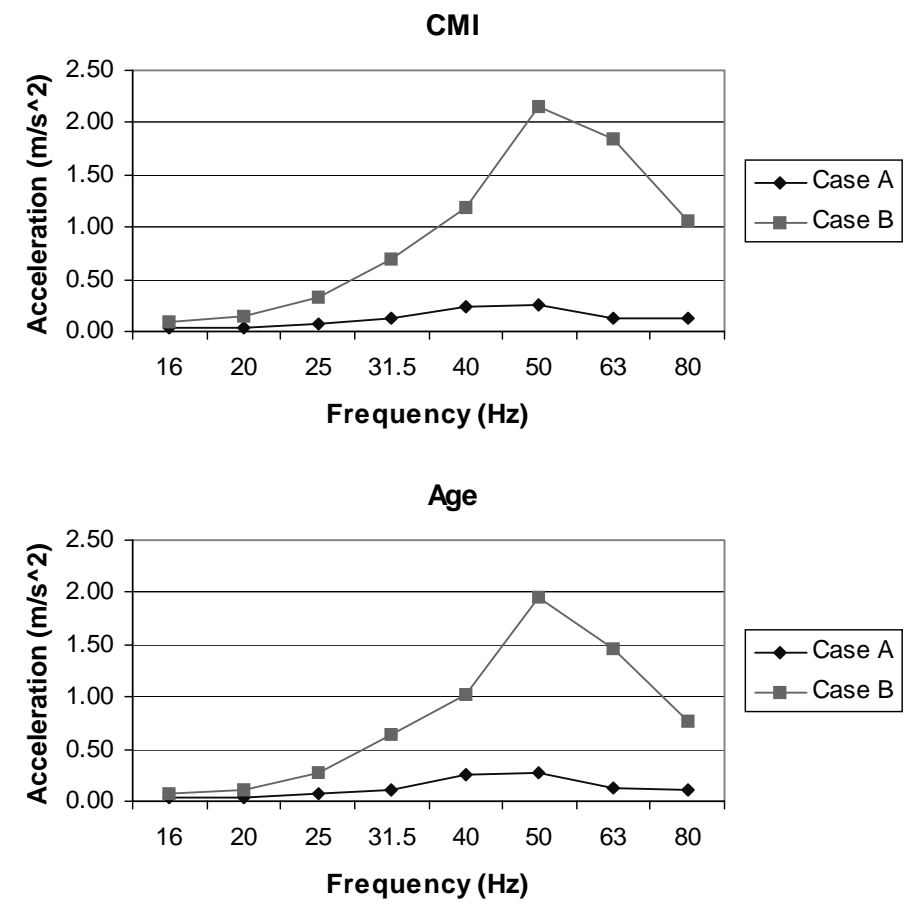

Vision

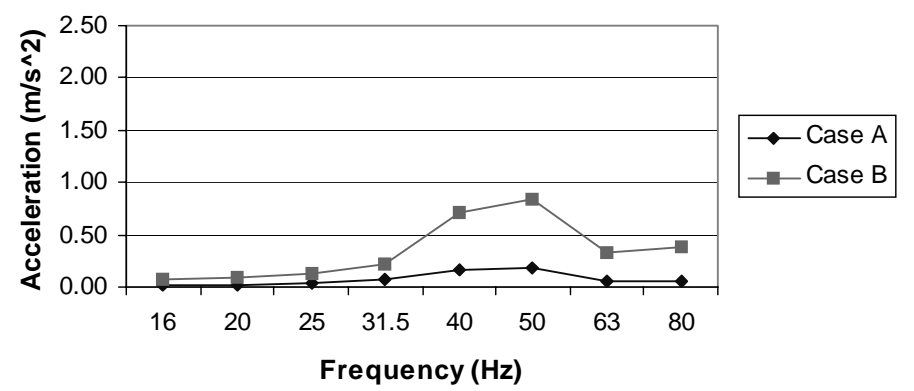

Fig. 8. Average results obtained for cases A and B when analyzing gender, CMI, age and vision.

studied presented the same curve behavior, i.e., the sensitivity decreased as the frequency increased. For case A (i.e., the perception threshold), regardless of the parameter being evaluated, all the results presented more or less the same values, even for vision (although the last one is slightly smaller than the others). For case B (i.e., maximum acceptable vibration at dwellings) the results for gender and age presented more or less the same sensitivities. The 
acceleration levels for CMI are slightly bigger than the others at most of the frequencies apart from $80 \mathrm{~Hz}$. Vision results, on the other hand, showed values much lower in general. It should be stressed that the volunteers that took part during the vision study were young in general and when age parameter was studied, they were more sensitive than the older group. This fact may have also influenced the results obtained during vision parameter studies, together with the previous knowledge of the tests.

\section{Conclusions}

This work evaluated the influence of the parameter vision in the vibration perception threshold and maximum acceptable vibration limit of subjects when exposed to some vibration at constructions, such as those using metallic structure. To that end, the results of two groups of volunteers were compared taking into consideration the extremes of the parameter in study (i.e., covered and uncovered eyes).

The experimental methodology used in this work was the same developed and used in [9] when studying the gender parameter. All volunteers were submitted to the same frequencies, to know, the center frequencies for the $1 / 3$ octave band in the range from 16 to $100 \mathrm{~Hz}$, due to the low frequency limit of the equipment used. The volunteers adjusted the acceleration amplitudes, although the frequency was presented in a random way by the researcher.

The vision results presented the same curve behavior found in previous studies considering gender, corporeal mass index (CMI) and age, i.e., the sensibility decreased with the increase of frequency (as acceleration level increased) reaching a minimum between $40 \mathrm{~Hz}$ and $50 \mathrm{~Hz}$ and later increased again at $63 \mathrm{~Hz}$, decreasing not as much as before at $80 \mathrm{~Hz}$. This fact indicates that independently of the parameter under investigation, the volunteers adjust small acceleration levels for lower frequencies, and high acceleration levels for higher frequencies, although for the series of studies performed here there is a decrease in the acceleration levels above $50 \mathrm{~Hz}$ again. Despite the same behavior, the values obtained for each parameter was similar for the perception threshold, although differ for the maximum acceptable vibration limit (mainly for vision).

Around 40 and $60 \mathrm{~Hz}$, the results may have been influenced by the resonant frequencies of the head and ocular globe. Also, at low frequencies, the setup used influenced the results obtained since there is vibration amplification at that region, making the subjects adjust smaller acceleration levels than at the high frequencies, where isolation occurs.

During the statistical analysis of the variable vision, no differences were found between the two groups studied. In spite of that, the average results for the covered eyes group was smaller than the average results for the uncovered eyes group. The fact of covering the eyes can be helping the subjects to be more concentrated, so making possible for them to notice the vibration of smaller intensity. However, the volunteers' results for the covered eyes could have being influenced by previous knowledge of the tests as well, as the same sample that took part previously in other tests were used for the analysis of the uncovered eyes. For most of the participants there was a decrease in the answers. No repeatability analysis was undertaken to confirm this fact. New tests shall be accomplished to confirm these hypotheses.

\section{Acknowledgments}

The authors wish to thank the volunteers who took part in the research and Marcos Roberto Misael and Luiz Eduardo de Assis Freitas Filho for the setting up of the experimental methodology adopted. They also wish to thank $\mathrm{CNPq}$ for the scholarship and FAPEMIG for funding some of the equipment used in this research.

\section{Responsibility notice}

The authors are the only persons responsible for the printed material included in this paper. 


\section{References}

[1] F.-K. Chang, Human response to motions in tall buildings, Journal of the Structural Division, American Society of Civil Engineers 99(ST6) (1973).

[2] M.L.M. Duarte, M.B. Pereira, M.R. Misael and L.E.A. Freitas Filho, Comparing Gender Influence against Age, Corporeal Mass Index and Vision on Comfort Levels to Whole-body Human Vibration, Proceedings of INTER-NOISE2005, CD-ROM, 2005, In05-2064.

[3] L.E.A. Freitas Filho, Studies of Human Comfort Levels to Vibration with Emphasis to the Modal Analysis of the Setup Used, in Portuguese, Final Year Report, Mechanical Engineering Department, Federal University of Minas Gerais, 2004.

[4] T.E. Fairley and M.J. Griffin, Predicting the discomfort caused by simultaneous vertical and fore-and-aft whole-body vibration, Journal of Sound and Vibration 124(1) (1988), 141-156.

[5] M.J. Griffin, Handbook of Human Vibration, Ed. Academic Press, 1996, 986.

[6] M.J. Griffin, A comparison of standardized methods for predicting the hazards of whole-body vibration and repeated shocks, JSV 215(4) (1998), 883-914.

[7] ISO 2631/1, Mechanical Vibration and Shock - Evaluation of Human exposure to Whole-Body Vibration - Part 1: General Requirements, 2nd Edition. Geneve, 1997.

[8] ISO 2631/2, Evaluation of Human exposure to Whole-Body Vibration - Part 2: Continuous and Shock-induced Vibration in Buildings (1 to $80 \mathrm{~Hz}) "$, 1st Edition. Geneve, 1989.

[9] M.R. Misael, Human Comfort to Vibration Levels, (In Portuguese), M.Sc. Dissertation, Mechanical Engineering Department, Federal University of Minas Gerais, Belo Horizonte/MG, Brazil, 2001.

[10] M.R. Misael, M.L.M. Duarte and L.E.A. Freitas Filho, Experimental Evaluation of Vibration Comfort for a Residencial Environment, IMAC XX, CD-ROM, 2002, 1376-1381.

[11] D.J. Oborne and P.A. Boarer, Subjective response to whole-body vibration: The effects of posture, Ergonomics 25 (1982), 637-681.

[12] D.J. Oborne and P.A. Boarer, Subjective response to whole-body vibration: The effects of instruction, Ergonomics 25 (1982), $759-769$.

[13] W.R. Paulino, Atual Biology, (Vol. 1), (In Portuguese), 13th Edition. São Paulo, Editora Ática, 1995.

[14] K.C. Parsons and M.J. Griffin, Whole-body vibration perception thresholds, JSV 121 (1988), $237-258$.

[15] M.B. Pereira and M.L.M. Duarte, Study of Parameters Affecting the Levels of Acceleration for Human Comfort, (In Portuguese), Proceedings of CONEM 2004, CD-ROM, 2004, 56028.

[16] C.C.G. Pereira, Human Perception and Comfort Curves to Vertical Vibration, (In Portuguese), M.Sc. Dissertation, Civil Engineering Department, Federal University of Ouro Preto, Ouro Preto/MG, Brazil, 2005.

[17] H. Seidel and R. Heide, Long-term effects of whole-body vibration: a critical survey of the literature, Intern. Architecture Occupation Environment Health $\mathbf{5 8}$ (1986), 1-26.

[18] M.R. Spiegel and D. Di Franco, Schaum's Outline of Theory and Problems of Statistics, Mc Graw-Hill, Inc. and Math Soft, Inc., 1997.

[19] H.E. Von Gierke, Biodynamic Models and Their Applications, Journal Acoustical Society of America 50(1397) (1971). Apud H.E. Von Gierke and A.J. Brammer, Shock and Vibration Handbook; Chapter 44: Effects of Shock and Vibration on Humans, (4th Edition), Cyril M. Harris.

[20] H.E. Von Gierke and A.J. Brammer, Shock and Vibration Handbook; Chapter 44: Effects of Shock and Vibration on Humans, (4th Edition), Cyril M. Harris, 1998. 

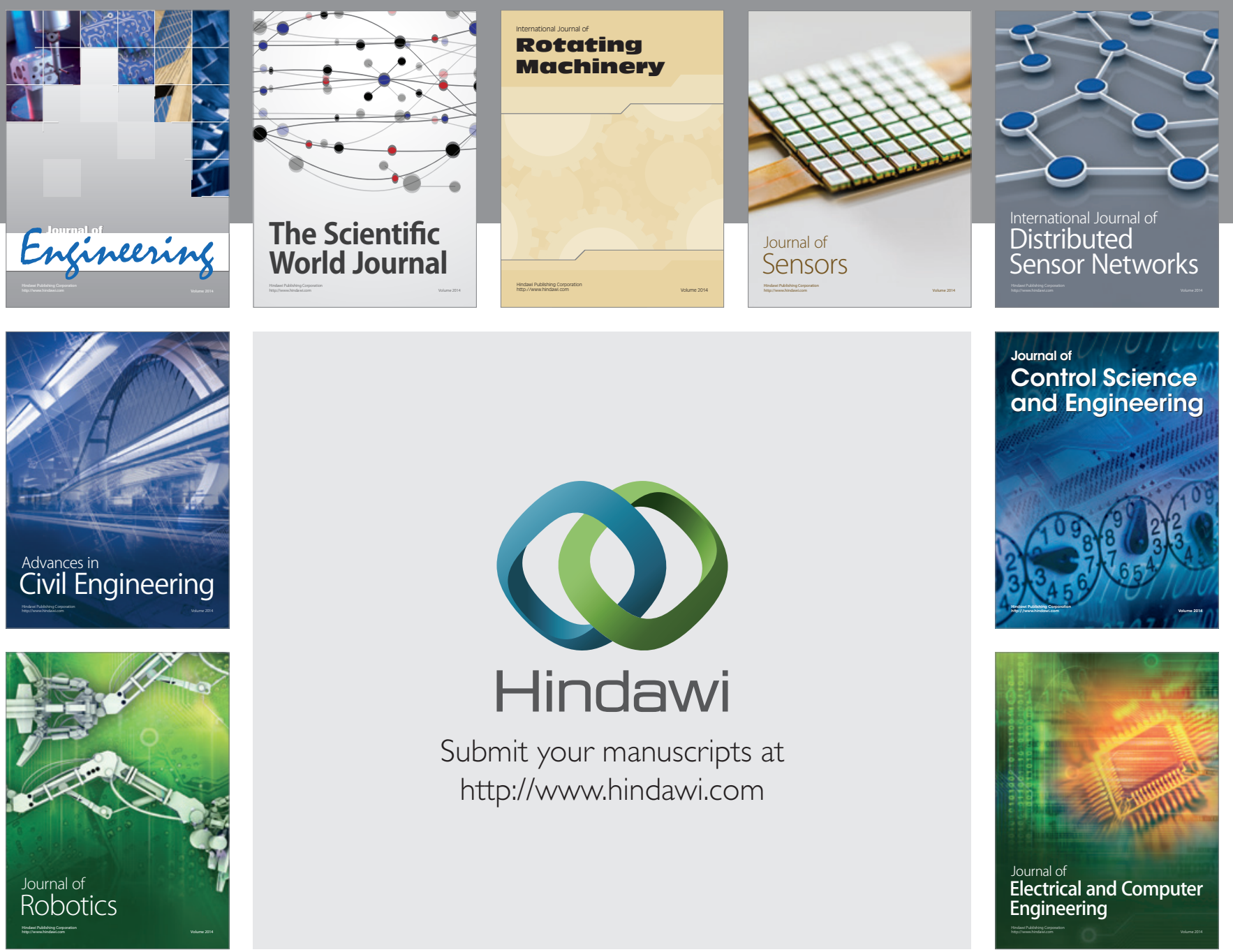

Submit your manuscripts at

http://www.hindawi.com
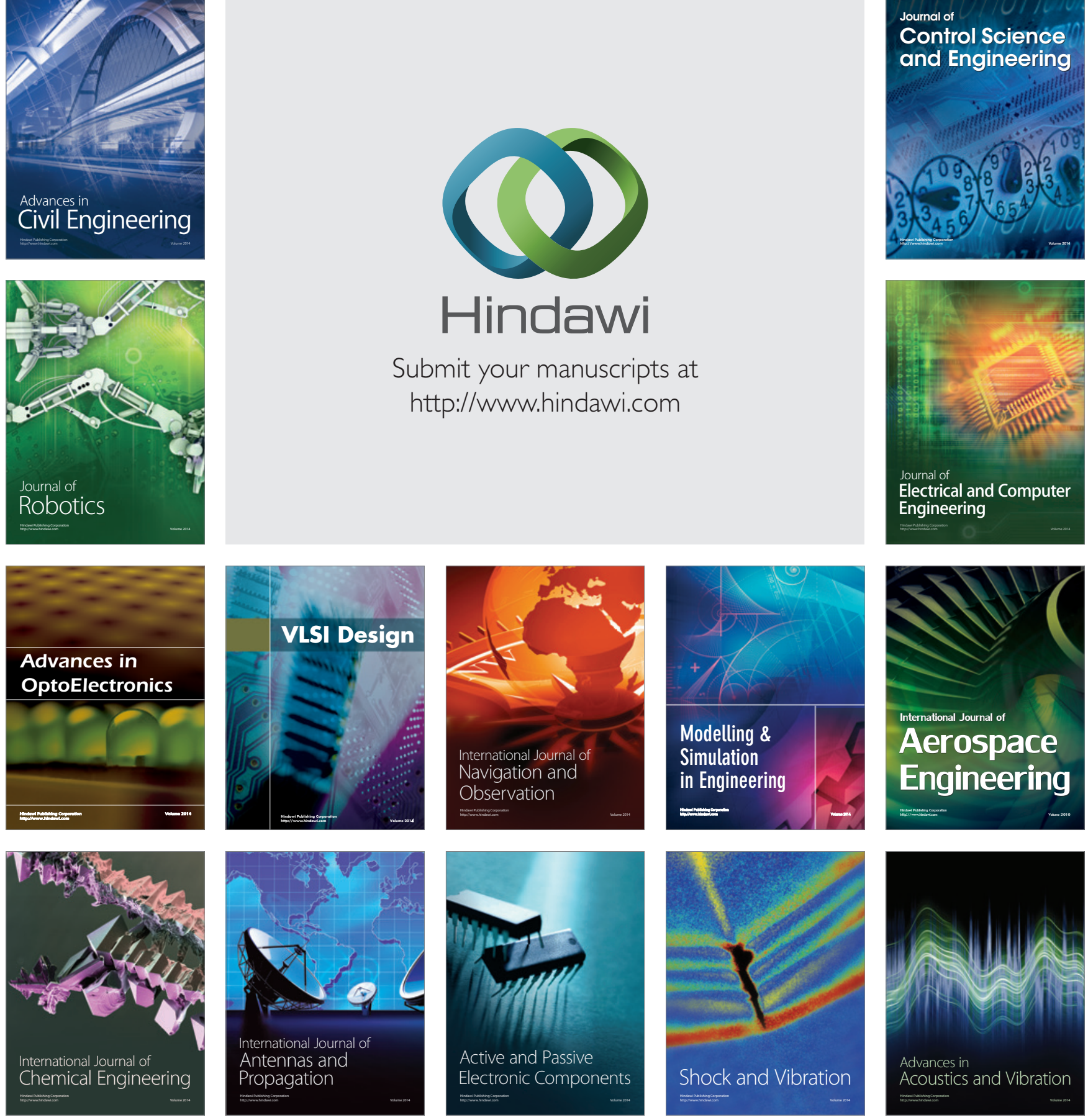